\title{
Pharmacoproteomics of obesity: definitions, role and a case study of dynamics of human
}

\section{plasma proteome}

\author{
"Pharmacoproteomics relates to pharmacogenomics as pharmacodynamics relates \\ to pharmacokinetics ... we can view pharmacoproteomics as the molecular \\ pharmacodynamics of the 'omics era."
}

\section{KEYWORDS: adipose tissue $\approx$ leptin $\approx$ leptin resistance $\approx$ obesity $\approx$ pharmacogenomics - pharmacoproteomics - plasma $\approx$ proteomics}

In this article, several definitions of pharmacoproteomics and why pharmacoproteomics are important for obesity studies are discussed. This illustrates with recent results on the dynamics of plasma proteome during leptin replacement therapy in genetically based leptin deficiency.

Pharmacoproteomics belongs to the third generation of the 'omics sciences. The first generation is represented solely by genomics, while the second includes proteomics and 21 other 'omes', as reflected in the OMES Table [101]. The term pharmacoproteomics is relatively new: it is still absent in the Oxford English Dictionary, which recognizes pharmacogenomics as "a branch of genomics dealing with the identification of genes involved in individual responses to drugs". A Scopus search for "pharmacoproteomics" returned 59 papers (the earliest published in 1999 [1]) and a PubMed search resulted in 43 papers. Interestingly, the Future Medicine Group recognized the importance of pharmacoproteomics early, which was mentioned in at least 18 publications in the group's journals and in five papers published in Pharmacogenomics, including $[2,3]$.

As is usually the case with new terms, the definition of pharmacoproteomics is not yet carved in stone. The president of the Human Proteome Organization wrote: "Pharmacoproteomics, a term that is a synthesis of 'pharmacology' and 'proteomics', refers to the comprehensive proteomics analysis that is relevant to the novel drug target discovery, drug metabolism, as well as drug efficacy and toxicity" [4]. Valdes et al. emphasized the role of pharmacoproteomics by noting that "variation in drug response is likely due to functional differences in critical proteins (absence, presence or modification of proteins) that influence the efficacy of the net drug response" and that "proteome related studies are also needed to better establish a functional basis for pharmacogenomics information" [2]. Alongside with the above complementary definitions, there exists a view that pharmacoproteomics is a synonym for chemical proteomics, that is, "the identification and characterization of direct small molecule-protein interactions" [3].

In agreement with the above statements, I prefer the broader definition of [4] with the addition that pharmacoproteomics relates to pharmacogenomics as pharmacodynamics relates to pharmacokinetics; the relation is famously and elegantly defined as follows: "Pharmacokinetics may be simply defined as what the body does to the drug, as opposed to pharmacodynamics which may be defined as what the drug does to the body" [5]. With this in mind, we can view pharmacoproteomics as the molecular pharmacodynamics of the 'omics era.

We can envision (following the logic of the OMES Table [101]) the pharmacoproteome as the combination of subpharmacoproteomes (sub-pp-omes), for example, aspirin-proteome, warfarin-proteome, simvastatin-proteome and so on; each of them being a population of proteins significantly altered (in abundance or modification) by a certain drug (not only those directly interacting with the drug, but all downstream of these interactions) and measured by comparison of proteomes after versus before. Obviously, each of these sub-pp-omes is subject to genetic variability, depends on the concentration of the drug, and is not independent of other sub-pp-omes. Luckily, some of these complications can be resolved with bioinformatics analysis, while others require additional measurements. It is useful to construct these sub-pp-omes not just as lists of proteins, but as networks that start with the proteins directly interacting with the drug, followed by the

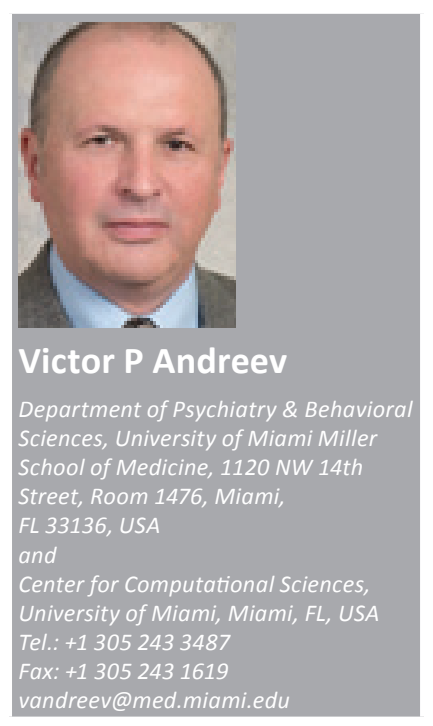

future micane $^{\text {pat }}$ fsg 
pathways reflecting both the drug's mechanism of action and the off-target effects. The important milestone in creating such networks has been achieved with the MetaDrug ${ }^{\mathrm{TM}}$ (GeneGo, St. Joseph, MI, USA).

'Omics sciences, including pharmacoproteomics, are especially important in understanding and treating common complex diseases, where the multiple genetic and environmental factors are intertwined [6]. Obesity is one such disease, where the role of environmental and lifestyle factors cannot be ignored, and therefore the dynamic, proteome-level measurements are important. Not surprisingly, a Scopus search for "proteomics" and "obesity" returned 321 papers, while "pharmacogenomics" and "obesity" resulted in 103 hits. However, the format of this article does not allow reviewing of these papers. An informative review is available, which provides a summary of the major findings and methodology of proteomic studies in obesity research [7]. Interestingly, of the 35 studies mentioned in this summary, 19 were performed with mice, six with rats and ten with humans. A total of 18 of these papers employed the 2D gel electrophoresis followed by matrix-assisted laser desorption ionization time-of-flight mass spectrometry analysis of the gel spots. Not arguing with the results of these important papers, it is worth mentioning that the $2 \mathrm{D}$ gel proteomics is now known to have major limitations owing to low resolution, low dynamic range and bias against the membrane proteins [8]. A total of 13 of the above 35 studies employed the state-ofthe-art shotgun proteomics approach. Among the important proteomics of obesity papers is the large-scale study of mouse adipocytes performed with nano liquid chromatography-tandem mass spectrometry, which resulted in identification of 3287 proteins [9].

The term 'pharmacoproteomics' is not found frequently in papers dealing with the proteomics of obesity papers. However, several of them do perform pharmacoproteomic studies, that is, compare proteomes before and after antiobesity treatments with: sibutramine [10], rosiglitazone [11,12], thiazolidinediones [13], chitosan oligosaccharides [14] and capsaicin [15]. The majority of these papers used the $2 \mathrm{D}$ gel electrophoresis approach $[10,12,14,15]$, while $[11,13]$ performed the state-of-the-art 2D liquid chromatography-tandem mass spectrometryquantitative proteomics with ${ }^{18} \mathrm{O}$ labeling. The important results include: increase in the level of proteins involved in GLUT4 transport in human adipose tissue after rosiglitazone treatment [12]; suppression of the obese adipose tissue secretome in rats after thiazolidinedione [13]; and downregulation of obesity related genes and normalization of altered proteins in ob/ob mice plasma by chitosan oligosaccharides [14].

Recently, I published a study on the dynamics of human plasma proteome during leptin replacement therapy [16]. Three unique adults with genetically caused leptin deficiency were treated and studied longitudinally during the course of recombinant human leptin replacement treatment (which started a decade ago). This resulted in the reversal of morbid obesity, resolved diabetes mellitus and hypogonadism, as described in [17].

\section{"It would be of value to integrate our results with the cross-sectional study of the proteomes of obese and nonobese humans stratified according to their leptin blood level."}

Leptin is an important regulator of food intake and energy homeostasis discovered in 1994 [18]. A rise in leptin level signals satiety to the brain through receptors in hypothalamic and brainstem neurons. Soon after an initial period of optimism about the possible treatment of obesity with exogenous leptin, it was observed that obese humans had high blood levels of leptin and that additional leptin treatment was unable to lead to weight loss in these patients, due to underlying leptin resistance [19]. The long-term goal of our proteomics study was to understand why the genetically deficient leptin patients did not develop resistance to the exogenous leptin, while nonleptin deficient obese patients did develop leptin resistance. The answer to this question might lead to the new, more efficient treatment of obesity.

Quantitative isobaric tag for relative and absolute quantitation labeled gel-free proteomics analysis was performed in plasma samples collected during four stages: before initiation of leptin treatment (leptin naive state), after 1.5 and 6 years of leptin-replacement therapy and after 7 weeks of interruption of the treatment. Approximately 500 proteins were reliably identified and quantitated in those four stages, 150 were 1.5 -fold or more differentially abundant. A total of 93 of these proteins (21 common for all patients) demonstrated synchronous dynamics of abundances, reflecting both short- and long-term effects of leptin replacement therapy. The bioinformatic analysis revealed that pathways and processes significantly enriched with the synchronous proteins were: cell adhesion, cytoskeleton remodeling, cell cycle, 
blood coagulation, glycolysis and gluconeogenesis. The transcription regulation network analysis revealed 17 plausible common regulators of the above synchronous proteins. This network included two transcription factors (c-Myc and the androgen receptor) known to activate each other through a double-positive feedback loop, which might stabilize the whole network in the 'on' state, and therefore, represent a molecular mechanism for the long-term effects of leptin replacement therapy.

Although interesting by themselves, these results alone do not answer the above question on whether it is possible to avoid leptin resistance in nonleptin deficient obese patients. Ideally, our results should be integrated with the results of comparative longitudinal study of proteomes from the patients starting from the initial nonobese state and then acquiring the state of obesity. More realistically, it would be of value to integrate our results with the cross-sectional study of the proteomes of obese and nonobese humans stratified according to their leptin blood level. Importantly, since we are interested in the not fully understood mechanism of leptin resistance, such study requires as broad and as deep as possible coverage of human proteome, and therefore should employ the state-of-the-art proteomics approaches and platforms.

Being a systemic disease, obesity requires a systems biology approach for its understanding. Proteomics and pharmacoproteomics should play an important role in the multidisciplinary effort to prevent, treat and reverse obesity. In the view of the worldwide epidemic of obesity and its consequences for healthcare and economy, it is worth the effort and expenses to launch the large-scale proteomics and pharmacoproteomics of obesity program employing the state-of-theart approaches based on high-resolution and high-throughput mass-spectrometry platforms.

\section{Financial \& competing interests disclosure}

The author has no relevant affiliations or financial involvement with any organization or entity with a financial interest in or financial conflict with the subject matter or materials discussed in the manuscript. This includes employment, consultancies, honoraria, stock ownership or options, expert testimony, grants or patents received or pending, or royalties.

No writing assistance was utilized in the production of this manuscript.

\section{Bibliography}

1 Moyses C. Pharmacogenetics, genomics, proteomics: the new frontiers in drug development. Int. J. Pharm. Med. 13(4), 197-202 (1999).

2 Valdes R Jr, Linder MW, Jortani SA. What is next in pharmacogenomics. Translating it to clinical practice. Pharmacogenomics 4(4), 499-505 (2003).

3 Kley N, Ivanov I, Meier-Ewert S. Genomic and proteomics tools for compound mode-of-action studies in drug discovery. Pharmacogenomics 5(4), 395-404 (2004).

4 Paik YK, Graham JE, Jone M, McDonald F, Holmes C, Ozdemir V. Interview with Dr. Young-Ki Paik, President of the Human Proteome Organization (HUPO): pharmacoproteomics and the approaching wave of 'proteomics diagnostics'. Curr. Pharmacogenomics Person. Med. 7, 243-248 (2009).

5 Benet LZ. Pharmacokinetics: basic principles and its use as a tool in drug metabolism. In: Drug Metabolism and Drug Toxicity. Mitchell JR, Horning MG (Eds). Raven Press, NY, USA (1984).

6 Relton CL, Smith GD. Epigenetic epidemiology of common complex disease: prospects for prediction, prevention, and treatment. PLoS Medicine 7(10), e1000356 (2010).
7 Barcelo-Batllori S, Gomis R. Proteomics in obesity research. Proteomics Clin. Appl. 3, 263-278 (2009).

8 Ong SE, Mann M. Mass spectrometry based proteomics turns quantitative. Nat. Chem. Biol. 5(2), 252-262 (2005).

9 Adachi J, Kumar C, Zhang Y, Mann M. In depth analysis of the adipocytes proteome by mass spectrometry and bioinformatics. Mol. Cell. Proteomics 6, 1257-1273 (2007).

10 Choi JW, Joo JI, Kim DH et al. Proteome changes in rat plasma in response to sibutramine. Proteomics 11, 1300-1312 (2011).

11 Hess S, Chen X. Applications of proteomics to the study of adipose tissue. Methods Mol. Biol. 456, 131-140 (2008).

12 Ahmed M, Neville MJ, Edelmann MJ, Kessler BM, Karpe F. Proteomic analysis of human adipose tissue after rosiglitazone treatment shows coordinated changes to promote glucose uptake. Obesity 18(1), 27-34 (2010).

13 Chen X, Hunt D, Cushman SW, Hess S. Proteomic characterization of thiazolidinedione regulation of obese adipose secretome in Zucker obese rats. Proteomics Clin. Appl. 3, 1099-1111 (2009).

14 Kumar SG, Rahman MA, Lee SH, Hwang HS, Kim HA, Yun JW. Plasma proteome analysis for anti-obesity and anti-diabetic potentials of chitosan oligosaccharides in ob/ob mice. Proteomics 9, 2149-2162 (2009).
15 Joo JJ, Kim DH, Choi JW, Yun JW. Proteomic analysis for antiobesity potential of capsaicin on white adipose tissue in rats fed with a high fat diet. J. Proteome Res. 9, 2977-2987 (2010).

16 Andreev VP, Dwivedi RC, Paz-Filho G et al. Dynamics of plasma proteome during leptin replacement therapy in genetically-based leptin deficiency. Pharmacogenomics J. 11, 174-190 (2011).

17 Licinio J, Caglayan S, Ozata M et al. Phenotypic effects of leptin replacement on morbid obesity, diabetes mellitus, hypogonadism, and behavior in leptin-deficient adults. Proc. Natl Acad. Sci. USA 101, 4531-4536 (2004).

18 Zhang Y, Proenca R, Maffei M, Barone M, Leopold L, Friedman JM. Positional cloning of the mouse obese gene and its human homologue. Nature 372(6505), 425-432 (1994).

19 Considine RV, Sinha MK, Heiman ML et al. Serum immunoreactive-leptin concentrations in normal-weight and obese humans. N. Engl. J. Med. 334, 292-295 (1996).

\section{Website}

101 OMES Table http://bioinfo.mbb.yale.edu/what-is-it/omes/ omes.html 